\title{
On the Envelope Correlation Coefficient of an LTE Reference Antenna System for Band 13
}

\author{
Bernhard Auinger, Michael Gadringer, Adam Tankielun, Christoph Gagern, and Wolfgang Bösch
}

\begin{abstract}
This document presents the investigations on two coupled reference antennas for LTE Band 13 (uplink 777MHz to $787 \mathrm{MHz}$, downlink $746 \mathrm{MHz}$ to $756 \mathrm{MHz}$ ). An important figure of merit to quantify coupling effects in multiple input multiple output (MIMO) antenna systems is the envelope correlation coefficient (ECC). The single antenna element is presented with radiation pattern and scattering parameters. It is a planar device, easy to be etched or milled as dimensions are given. The ECC investigations were done by measurement and simulation, the results are presented and compared. A good agreement between the simulated and the measured ECC can be recognized.
\end{abstract}

Index Terms-Band 13, ECC, envelope correlation coefficient, LTE reference antenna, MIMO antennas.

\section{INTRODUCTION}

In wireless data transmission the use of Multiple Input Multiple Output (MIMO) antenna technology introduces yet another degree of freedom and vastly rises the reachable data throughput. Transmission systems take advantage of the several different propagation paths. They send different data streams through multiple antennas on different paths through the wireless channels. Receivers pick up the mixed data streams. By orthogonalization in the receiver these paths can be treated as separated ones. The less the received data are correlated, the higher is the performance of the transmission system. Simply spoken, unnecessary introduction of data correlation has to be avoided in all stages of the transmission.

Looking at small sized hand held user equipment (UE) it is likely that antennas are closely spaced to each other. This proximity introduces unwanted coupling effects and in this way correlation, which results in a performance degradation. [1]-[15] perform similar investigations, but deal with antennas for other purposes. This paper shows an investigation of the ECC on an antenna setup with two coupled dipoles for LTE band 13.

\section{THEORETICAL BACKGROUND}

The Envelope Correlation Coefficient (ECC) $\rho_{\mathrm{e}}$ is an important figure of merit for the comparison of MIMO capabilities of coupled antennas. It was originally suggested by [16]. The following considerations are for an antenna system with two antennas. The incident E-field $\boldsymbol{F}$ consists of two polarization parts. $\Theta$ (zenith) and $\Phi$ (azimuth) denote the angles of certain direction in the spherical system, $\boldsymbol{i}_{\Theta}$ and $\boldsymbol{i}_{\Phi}$

Manuscript received September 29, 2014; revised November 30, 2014

The authors are with the Graz University of Technology, Austria (e-mail Bernhard.auinger@tugraz.at). are spherical unit vectors [17].

$$
\boldsymbol{F}(\Phi, \Theta)=\boldsymbol{F}_{\Phi}(\Phi, \Theta) \boldsymbol{i}_{\Theta}+\boldsymbol{F}_{\Theta}(\Phi, \Theta) \boldsymbol{i}_{\Theta}
$$

Two random incoming E-field vectors of a certain polarization are assumed to be uncorrelated

$$
\begin{aligned}
& E\left\{F_{v}(\Omega) F_{v}(\Omega)^{*}\right\}=0 \\
& v \in[\Phi, \Theta]
\end{aligned}
$$

The complex E-Field antenna pattern for the two antennas $E_{1}(\Omega)$ and $E_{2}(\Omega)$ also consist of $\Theta$ and $\Phi$ polarized parts

$$
\boldsymbol{E}_{k}(\Phi, \Theta)=E_{\Phi, k}(\Phi, \Theta) \boldsymbol{i}_{\Theta}+E_{\Phi, k}(\Phi, \Theta) \boldsymbol{i}_{\Phi}
$$

The antennas have to have the same phase reference [16]. The observed signals at the antenna ports $V_{1}$ and $V_{2}$ result from the incident field $\boldsymbol{F}(\Omega)$ hitting the complex antenna E-field pattern $\boldsymbol{E}_{1}(\Omega)$ and $\boldsymbol{E}_{2}(\Omega)$

$$
\begin{aligned}
& V_{1}(\Omega)=\oint \boldsymbol{E}_{1}(\Omega) \bullet \boldsymbol{F}(\Omega) d \Omega \\
& V_{2}(\Omega)=\oint \boldsymbol{E}_{2}(\Omega) \bullet \boldsymbol{F}(\Omega) d \Omega
\end{aligned}
$$

The symbol - denotes the Hermitian product, ${ }^{*}$ is the conjugate complex.

Simply spoken, every incoming E-field is received with the antenna gain for a certain $\Omega$ and vice versa. $\Omega(\Theta, \Phi)$ indicates the spherical angle constellation in zenith $(\Theta)$ and azimuth $(\Phi)$. The spherical integrand $d \Omega$ expands to

$$
\oint d \Omega=\int_{0}^{2 \pi} \int_{0}^{\pi} \sin \Theta d \Theta d \Omega
$$

Doppler shift and movement of the antennas have been neglected. The cross polarization rate XPR is defined as the ratio $P_{\Theta} / P_{\Phi} . P_{\Theta}$ and $P_{\Phi}$ are the mean received powers in $\Theta$ and $\Phi$ polarizations over all positions $\Omega(\Theta, \Phi)$. Calculating the cross correlation of the received signal $V_{1}$ and $V_{2}$ leads to

$$
\begin{gathered}
R_{12}=E\left\{V_{1} V_{2}^{*}\right\}=P_{\Phi} \oint X P R \cdot E_{\Phi 1}(\Omega) E_{\Phi 2}^{*}(\Omega) p d f_{\Theta}(\Omega)+ \\
E_{\Phi 1}(\Omega) E_{\Phi 2}^{*}(\Omega) \operatorname{pdf}_{\Phi}(\Omega) d \Omega
\end{gathered}
$$

$p d f_{\Theta}(\Theta, \Phi)$ and $\operatorname{pdf}_{\Phi}(\Theta, \Phi)$ are the angular density functions of the incoming electromagnetic waves. They fulfill the property

$$
\oint \operatorname{pdf}_{\Theta}(\Phi, \Theta) d \Omega=\oint \operatorname{pdf}_{\Theta}(\Phi, \Theta) d \Omega=1
$$

Assuming that the incoming signal $V_{1}$ and $V_{2}$ are Gaussian 
distributed and have zero mean value, the variances are $(m \in$ $\{1,2\}$ )

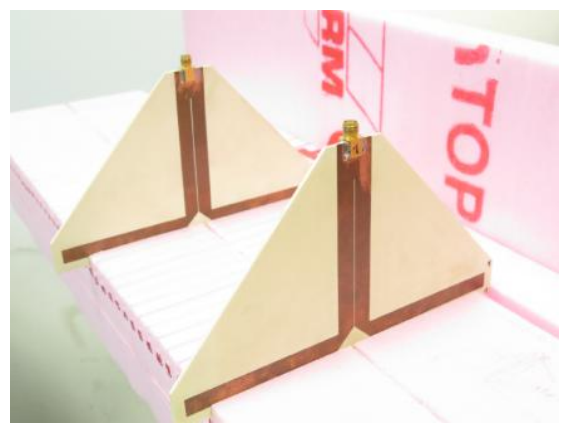

Fig. 1. Setup for the measurement of the antenna system.

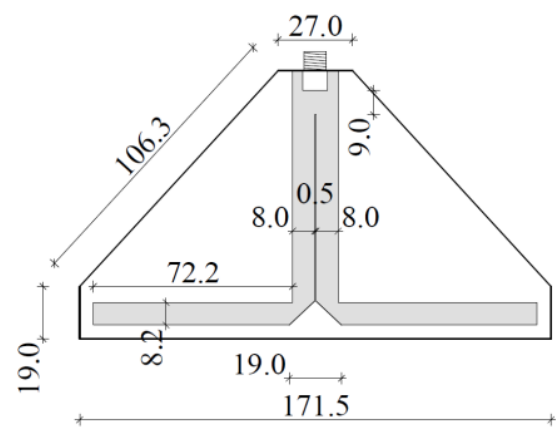

Fig. 2. Dipole and transformer.

$$
\begin{gathered}
\sigma_{m}^{2}=E\left\{V_{m} V_{m}^{*}\right\}=P_{\Theta} \oint \operatorname{XPR} \cdot E_{\Theta, m}(\Omega) E_{\Theta, m}^{*}(\Omega) \operatorname{pdf}_{\Theta}(\Omega)+ \\
E_{\Phi, m}(\Omega) E_{\Phi, m}^{*}(\Omega) \operatorname{pdf}_{\Phi}(\Omega) d \Omega
\end{gathered}
$$

The Envelope Correlation Coefficient (ECC) $\rho_{\mathrm{e}}$ is now calculated by the squared absolute cross correlation of the received signals divided by the product of their single variances [18].

$$
\sigma_{e} \cong \frac{\left|R_{12}\right|^{2}}{\sigma_{1}^{2} \sigma_{2}^{2}}
$$

However, calculating $\rho_{e}$ this way needs lots of measurement effort, because the antenna system has to be measured over the whole sphere to get the complete picture.

Diallo et al found a simplified method [19], [20]. They use the reciprocity of antennas and found an equivalence between the complex E-field pattern and the S-parameters of the antenna system under certain circumstances. This leads to a formula, where the $\rho_{\mathrm{e}}$ can be calculated by means of the scattering parameters of the antenna system.

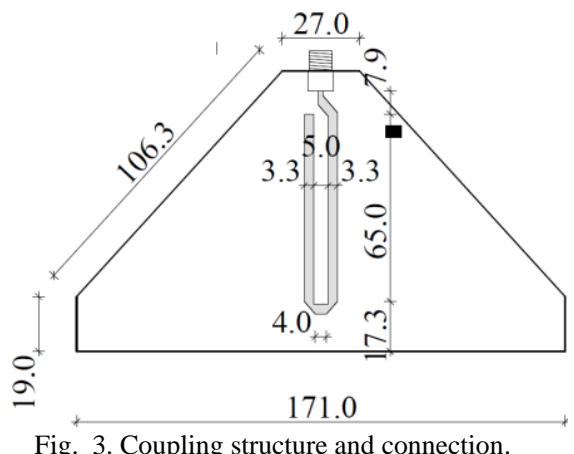

Fig. 3. Coupling structure and connection.
Vector Network Analyzer

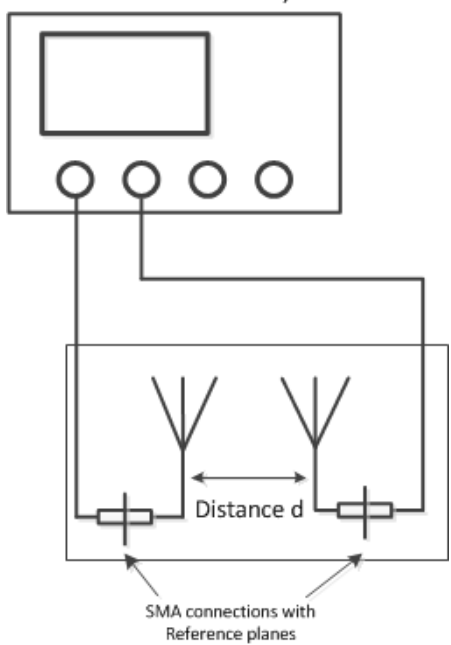

Fig. 4. Measurement setup of the antenna group.

$$
\sigma_{e} \cong \frac{\left|S_{11}^{*} S_{12}+S_{21}^{*} S_{22}\right|^{2}}{\left(1-\left(\left|S_{11}\right|^{2}+\left|S_{21}\right|^{2}\right)\right)\left(1-\left(\left|S_{22}\right|^{2}+\left|S_{12}\right|^{2}\right)\right)}
$$

Within certain limitations this simplified computation delivers good comparisons possibilities between antenna systems [19]:

- Antennas are assumed to be lossless

- Antennas are placed in a uniform multipath environment, which is an ideal case

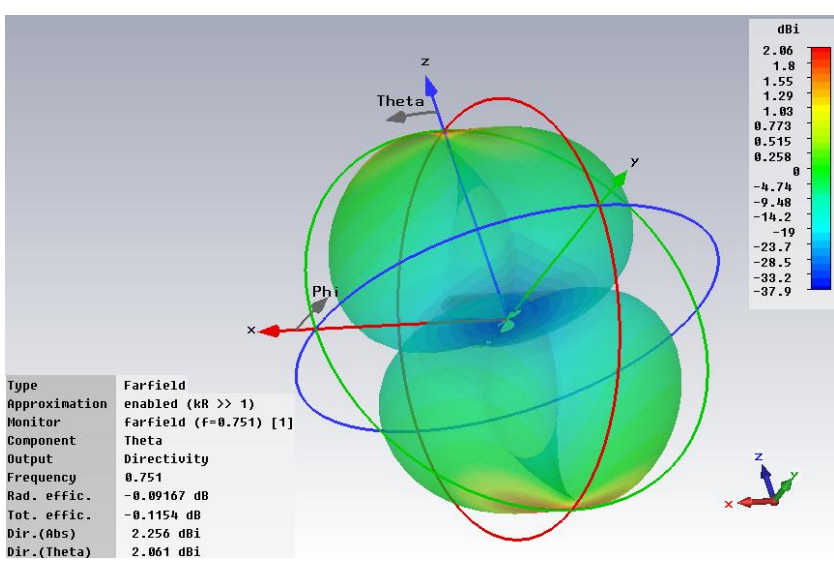

Fig. 5. Directivity of the single antenna in $\Theta$ polarization.

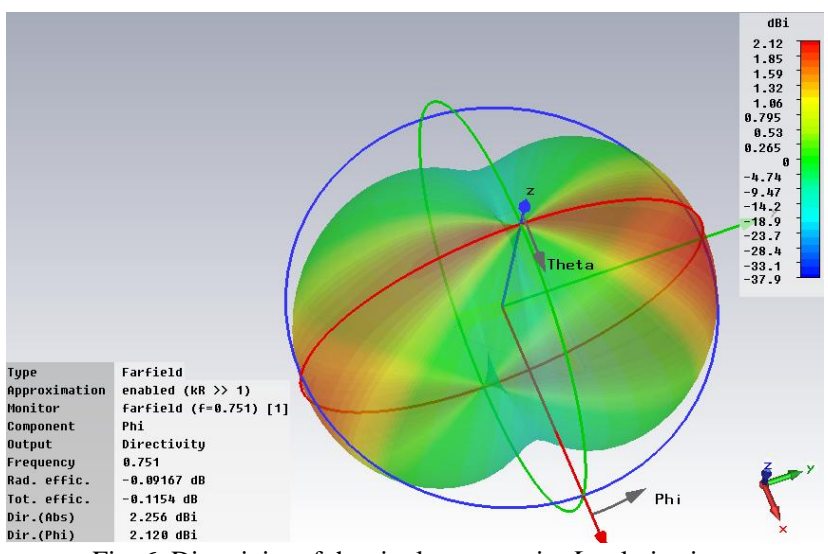

Fig. 6. Directivity of the single antenna in $\Phi$ polarization.

\section{REFERENCE ANTENNAS AND SETUP}

A practical antenna system containing two reference 
antennas for LTE band 13 (Fig. 1) has been investigated. Antenna parameters have been simulated and measured.

The antenna is manufactured on a printed circuit board (PCB) with Rogers 4350B substrate characterized by an $\varepsilon_{r}=$ 3.48. The employed test antennas were milled on the PCBs. The antenna is a resonant antenna and designed for the LTE Band 13, with downlink frequency range from $746 \mathrm{MHz}$ to $756 \mathrm{MHz}$, and uplink range from $777 \mathrm{MHz}$ to $787 \mathrm{MHz}$.

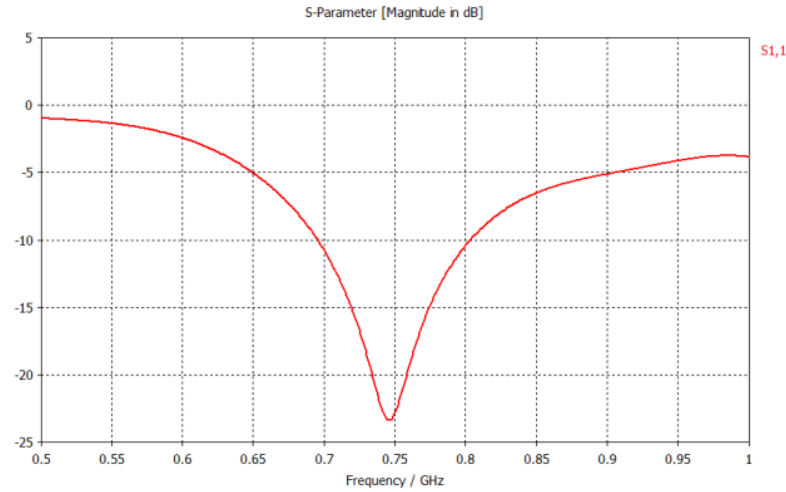

Fig. 7. Abs. refl. coefficient $S_{11}$ of single ref. antenna in $\mathrm{dB}$.

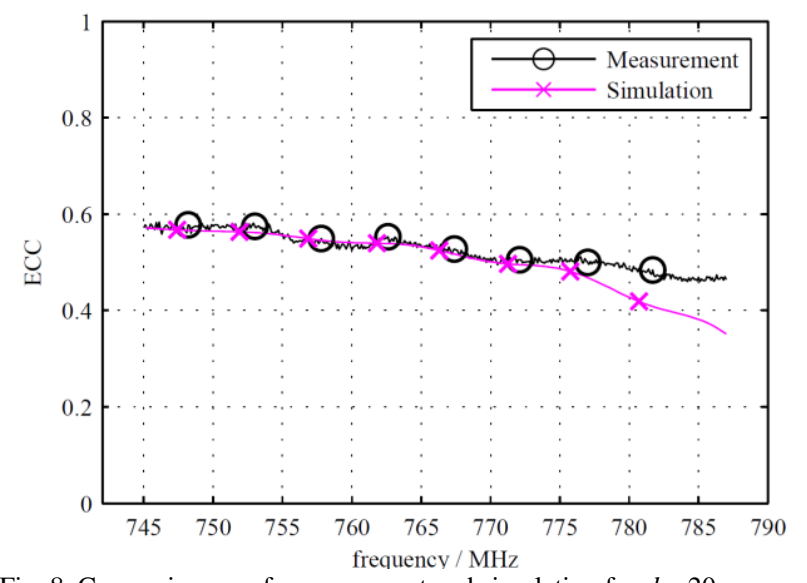

Fig. 8. Comparison $\rho_{\mathrm{e}}$ of measurement and simulation for $d=20 \mathrm{~mm}$.

The utilized antenna system consists of two single antennas, Fig. 1 shows the setup in reality. Each antenna is composed of a dipole and a $\lambda / 4$ transformer (Fig. 2) located on one side of the printed circuit board (PCB). On the other side a coupling structure (Fig. 3), which also serves a BALUN (Balanced Unbalanced transformer) is placed. It minimizes the influence of the cable. The simulated directivities of a single antenna are indicated in Fig. $5(\Theta$ polarization) and Fig. 6 ( $\Phi$ polarization).

The reflection coefficient $S_{11}$ of a single reference antenna is shown in Fig. 7. It is always below than $-12 \mathrm{~dB}$ in the specified frequency range. If the user is willing to accept a certain degradation in reflection coefficient $\left(S_{11}\right.$ always below $-10 \mathrm{~dB}$ ), the reference antenna system is also capable for the use with LTE Band 12 (downlink $729 \mathrm{MHz}$ to $746 \mathrm{MHz}$, uplink $699 \mathrm{MHz}$ to $716 \mathrm{MHz}$ ) and band 14 (downlink $758 \mathrm{MHz}$ to $768 \mathrm{MHz}$, uplink $788 \mathrm{MHz}$ to $798 \mathrm{MHz}$ ). Nevertheless, investigations have been done for Band 13.

The antenna system is placed with a polystyrene stand, with placing slots for changing the distance between the antennas. Polystyrene has a dielectric constant $\varepsilon_{r}$ close to one. This results in a very low influence of the stand on the results.
All measurements have been accomplished in an anechoic chamber, to avoid influence of laboratory facilities and equipment.

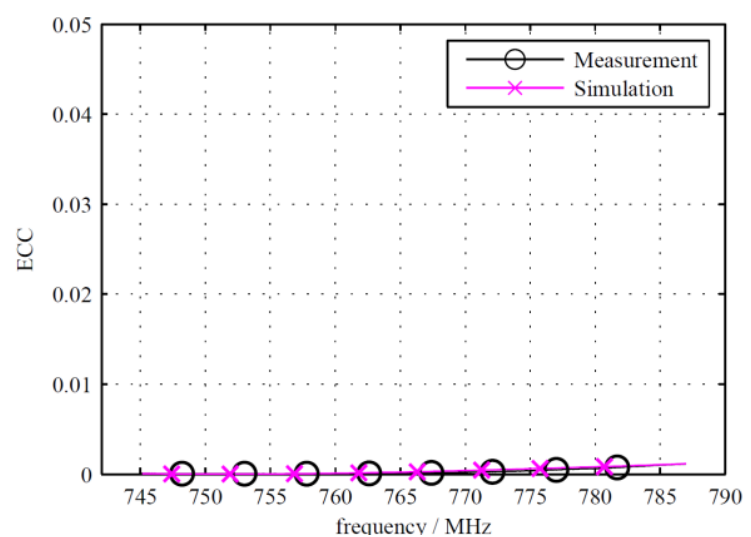

Fig. 9. Comparison $\rho_{\mathrm{e}}$ of measurement and simulation for $d=300 \mathrm{~mm}$.

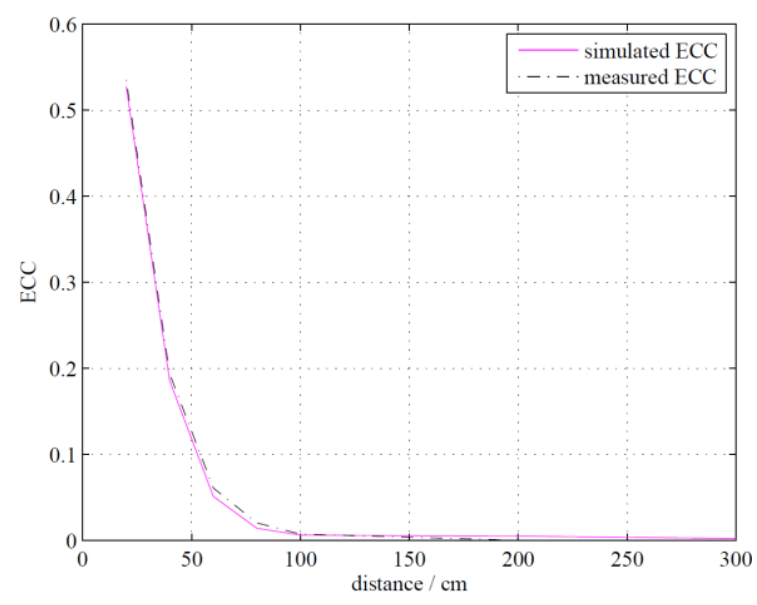

Fig. 10. ECC $\rho_{\mathrm{e}}$ over distance at $766 \mathrm{MHz}$.

\section{INVESTIGATIONS}

A parallel antenna setup has been chosen for the antenna stand setup (Fig. 1), because highest possible coupling of the antennas was expected in this case. The distance used were $20 \mathrm{~mm}, 40 \mathrm{~mm}, 60 \mathrm{~mm}, 80 \mathrm{~mm}, 100 \mathrm{~mm}, 200 \mathrm{~mm}$ and $300 \mathrm{~mm}$. Both antennas have the same orientation with the dipoles aligned in the same direction. The ECC $\rho_{\mathrm{e}}$ has been determined using both the measured and the simulated $\mathrm{S}$-parameters. This task was performed over the specified range of distances between the antennas.

\section{A. Simulation Setup}

Simulations have been done in CST Microwave Studio. The antennas have been placed in air the same way as applied in the measurements.

\section{B. Measurement Setup}

The S-parameters have been measured using coaxial cable connections to two ports of the vector network analyzer. Calibration of the measurement setup was accomplished up to the male SMA connectors of the connection cables, as depicted in Fig. 4.

\section{Discussion of Simulated and Measured Results}

The results are presented in two different scalings. The diagrams for $2 \mathrm{~cm}$ (Fig. 8) and $6 \mathrm{~cm}$ (Fig. 9) use the full possible ECC $\rho_{\mathrm{e}}$ scaling from zero to one. 
The diagrams for $200 \mathrm{~mm}$ (Fig. 10) and 300mm distance (Fig. 11) employ a scaling from 0 to 0.05 , representing the lower $\rho_{\mathrm{e}}$ for bigger distances $d$.

The results obtained in the investigations, both simulation and measurement, show a clear trend of a decreasing ECC $\rho_{\mathrm{e}}$ with increasing distance between the coupled antennas.

The simulation result show a slightly faster decrease of the ECC $\rho_{\mathrm{e}}$ than the measurement data for small distances $(d<$ $60 \mathrm{~mm}$ ). This effect is vanishing for bigger antenna distances.

The influence of the distance on the ECC $\rho_{\mathrm{e}}$ can be seen in Fig. 12 for $760 \mathrm{MHz}$. The investigations confirm the expected behaviour of a shrinking Envelope Correlation Coefficient $\rho_{\mathrm{e}}$ with bigger distances.

As mentioned, the chosen S-parameter method for calculation of the ECC $\rho_{\mathrm{e}}$ is ascertained to give a fast estimation on the quality of a MIMO antenna system. There is good congruence between measurement and simulation results.

\section{CONCLUSION}

In this paper the theory of the Envelope Correlation Coefficient (ECC) has been presented. It is a good figure of merit for comparing coupled MIMO antenna systems. It is applicable with a vector network analyzer only, no base station equipment is necessary.

A single LTE reference antenna for Band 13 with its physical appearance and directivity pattern is indicated. A system employing two of these antennas has been investigated.

There are two possible ways of achieving the ECC $\rho_{\mathrm{e}}$, the one using S-parameters has been chosen. The ECC is decreasing with increasing distance of the coupled antennas. Investigation results are presented for simulation and measurement. Good accordance between both is given.

\section{FUTURE WORK}

Future investigations should be done on the relationship between the ECC of an LTE transmission system and its achievable throughput. It is also intended to show the relationship of the condition number of a transmission matrix and the ECC by using S-parameters.

Also different placement of the antennas with weaker coupling should be investigated.

\section{REFERENCES}

[1] G. J. Foschini and M. J. Gans, "On limits of wireless communications in a fading environment when using multiple antennas," Wireless Pers. Comm., vol. 6, no. 3, pp. 311-335, March 1998.

[2] S. M. Ali and J. Warden, "Controlling coupling between two transmitting antennas for MIMO handset applications," Personal Indoor and Mobile Radio Communications (PIMRC), pp. 11-14, 2011

[3] H. Arun, A. K. Sarma, M. Kanagasabai, S. Velan, C. Raviteja, and M. G. N. Alsath, "Deployment of modified serpentine structure for mutual coupling reduction in MIMO antennas," Antennas and Wireless Propagation Letters, vol.13, pp. 277-280, 2014.

S. Zhang, A. A. Glazunov, Z. Ying, and S. He, "Reduction of the envelope correlation coefficient with improved total efficiency for mobile LTE MIMO antenna arrays: Mutual scattering mode," Antennas and Propagation, vol. 61, no. 6, pp. 3280-3291, June 2013.

[4] H. S. Sing, G. K. Pandey, P. K. Bharti, and M. K. Meshram, "Compact penta-band coupled-fed printed monopole MIMO/diversity antenna for smart mobile phone," Microwave and Photonics, pp. 1-5, 13-15, 2013.
[5] C. S. Chuang, W. T. Hsu, and L. M. Chun, "A compact dual band tree-type MIMO antenna for mobile wireless access network application," in Proc. the Microwave Conference Proceedings (APMC), 2013, pp. 1169-1171.

[6] S. Seymour, "On cross coupling in multiple-beam antennas," IRE Transactions on Antennas and Propagation, vol. 10, no. 5, pp. 548-557, September 1962

[7] S. W. Lee, A. E. Fathy, S. M. Ghazaly, and V. Nair, "Evaluation of optimum position and orientation of laptop MIMO antennas using envelope correlation coefficients and mutual coupling parameters," in Proc. the Antennas and Propagation Society International Symposium, vol. 2, 2007.

[8] V. Sorin, A. Artemenko, A. Sevastyanov, and R. Maslennikov, "Compact 2.5 to $2.7 \mathrm{GHz}$ two element MIMO antenna system for modern USB dongle," in Proc. the 6th European Conference on Antennas and Propagation (EUCAP), 2012, pp. 1955-1959.

[9] G. Srinivas, D. Jabin, and A. K. Singh, "Multiband MIMO antenna with reduction in mutual coupling and ECC," in Proc. the Students Conference on Engineering and Systems (SCES), pp. 1-5, 2014.

[10] W. S. Chen, K. M. Lin, B. Y. Lee, and C. L. Ciou, "A separated cross-shaped isolation element for WLAN MIMO applications," in Proc. the Microwave Conference Proceedings (APMC), 2013, pp. 1091-1093.

[11] L. Zhanmeng, L. Chunlan, Y. Luqu, J. Jianxin, and Y. Jie, "A novel compact dual-band MIMO antenna for WLAN application," in Proc. the International Conference on Microwave and Millimeter Wave Technology (ICMMT) 2012, 2012, vol. 3, pp. 1-4.

[12] M. A. H. Eleiwa, "Correlation characteristics of diversity antennas in mobile environments," in Proc. the 6th International Conference on Communications and Signal Processing, 2007, pp. 1-4.

[13] X. Zhou, X. L. Quan, and R. L. Li, "A dual-broadband MIMO antenna system for GSM/Umts/LTE and WLAN handsets," Antennas and Wireless Propagation Letters, vol.11, pp. 551-554, 2012.

[14] Q. Wang, D. Plettemeier, H. Zhang, K. Wolf, and E. Ohlmer, "A Diversity performance of an optimized meander PIFA array for MIMO handsets," Antennas and Wireless Propagation Letters, vol. 11, pp. 551-554, 2012.

[15] T. Taga, "Analysis of correlation characteristics of antenna Diversity in land mobile radio environments," Electronics and Communications in Japan, vol. 74, no. 8, 1991

[16] J. D. Kraus and R. J. Marhefka, Antennas: for All Applications, McGraw-Hill, New York 2003.

[17] R. H. Clarke, "A statistical theory of mobile radio reception," Bell Syst. Tech. Journal, pp. 957-1000, 1968.

[18] A. Diallo, P. L. Thuc, C. Luxey, R. Staraj, G. Kossiavas, M. Franzen, and P. S. Kildal, "Diversity characterization of optimized two-Antenna systems for UMTS handsets," EURASIP Journal on Wireless Communications and Networking, vol. 2, 2007.

[19] S. Blanch, J. Romeo, and I. Corbella, "Exact representation of antenna system diversity performance from input parameter description," Journal on Wireless Communications and Networking, vol. 3, 2007.

Bernhard Auinger received his master degree in electrical engineering (Dipl. Ing.) from Graz University of Technology in 2004. From 2005 to 2011 he initiated and ramped up electromagnetic compatibility pre-compliance tests for automotive ICs at Philips Semiconductors and NXP Semiconductors. Since 2011, he is an assistant at the Institute of Microwave and Photonic Engineering at the Graz University of Technology.

He was engaged in the comet mission ROMAP/ROSETTA mission of the European Space Agency. His current research interests include theoretical investigations on test procedures for MIMO enabled user equipment.

Michael Gadringer received his Dipl.-Ing. and the Dr. techn. degrees from the Vienna University of Technology in 2002 and 2012, respectively. Since 2010 he is an assistant at the Institute of Microwave and Photonic Engineering at the Graz University of Technology. During his studies he was involved in the design of analog and digital linearization systems for power amplifiers and with the behavioral modeling of microwave circuits. His current research interests include design and linearization of broadband microwave and mm-wave communication systems as well as on measurement techniques and de-embedding. He has co-edited the book "RF power amplifier behavioral modeling" published by Cambridge University Press.

Adam Tankielun received his mgr inż. and Dr.-Ing. degrees in electrical engineering from the Technical University of Gdańsk in 2002 and the Leibniz University of Hannover in 2007, respectively. From 2003 to 2008, he developed electromagnetic near-field scanning techniques for the Fraunhofer Institute for Reliability and Microintegration in Paderborn. Since 2008 he has been with ROHDE and SCHWARZ GmbH \& Co. KG in 
Munich developing over-the-air test systems for wireless devices.

Wolfgang Bösch has joined the Graz University of Technology in Austria to establish a new Institute for Microwave and Photonic Engineering. Prior he has been the CTO of the advanced digital institute in the UK, a non-profit organization to promote research activities. He has also been the director of Business and Technology Integration of RFMD UK. For almost 10 years he has been with Filtronic plc as CTO of Filtronic Integrated Products and director of the Global Technology Group. Prior to joining Filtronic, he held positions in the european space agency (ESA) working on amplifier linearization techniques, MPR-Teltech in Canada working on MMIC technology projects and the Corporate R\&D group of M/A-COM in Boston where he worked on advanced topologies for high efficiency power amplifiers. For four years he was with Daimler Chrysler Aerospace in Germany, working on T/R Modules for airborne radar.

Dr. Bösch received his engineering degrees from the Technical University of Vienna and Graz in Austria. He finalized his MBA with distinction at Bradford University School of Management in 2004. He is a fellow of the IEEE and a Fellow of the IET. He published more than 50 papers and holds 4 patents. He was a non-executive director of Diamond Microwave Devices (DMD) and is currently a non-executive director of the Advanced Digital Institute (ADI) and VIPER-RF company. 